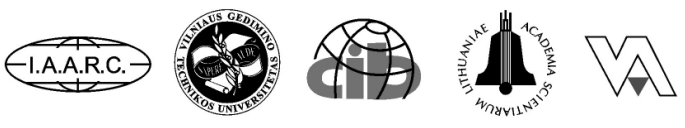

Institute of Internet and Intelligent Technologies

Vilnius Gediminas Technical University

Sauletekio al. 11, 10223 Vilnius, Lithuania

http://www.isarc2008.vgtu.lt/

\author{
The $25^{\text {th }}$ International Symposium \\ on Automation and Robotics in Construction
}

June 26-29, 2008

ISARC-2008

\title{
MULTIPLE-CRITERIA INTERACTIVE PROCEDURE IN PROJECT MANAGEMENT
}

\author{
Tomasz Błaszczyk \\ The Karol Adamiecki University of Economics \\ ul. 1 Maja 50, 40-287 Katowice \\ Email: blaszczyk@ae.katowice.pl
}

\author{
Maciej Nowak \\ The Karol Adamiecki University of Economics \\ ul. 1 Maja 50, 40-287 Katowice \\ Email: nomac@ae.katowice.pl
}

\begin{abstract}
While constructing project's schedule three criteria must be considered: time, cost, and scope. Additionally risk related to all that criteria also has to be taken into account. Thus, project planning problem can be defined as a multiple-criteria decision problem under risk. In the paper a project scheduling problem is analyzed. We assume, that various alternative resources allocations can be considered. The decision maker must determine the alternative acceptable with respect to cost and time. A new technique based on computer simulation and interactive approach is proposed. In the first step, simulation experiments are performed to evaluate decision alternatives with respect to criteria. The results are used for constructing distributional evaluations of alternatives. An interactive technique STEP-DPR is employed for generating the final solution of the problem. The procedure uses stochastic dominance and almost-stochastic dominance rules for comparing decision alternatives with respect to criteria. In each iteration a proposal solution is identified. This solution is presented to the decision maker. If he/she is satisfied with the solution - the procedure ends, otherwise the decision maker is asked to provide the information which can be used to improve the solution. A numerical example is presented to illustrate the applicability of the proposed technique.
\end{abstract}

\section{KEYWORDS}

Project planning, Multi-criteria decision aiding, Interactive approach, Simulation

\section{INTRODUCTION}

As far as projects are concerned, one of their specific aspects is the current need to make decisions which will result in an unpredictable state in the future. Project systematic nature requires each decision analysis concerning selected objects in project system to take into account all interactions with other objects and, eventually, the change of their parameters. Several objects may be characterized by multiple parameters which may influence the attributes of the whole project on different level. One the examples is a link between time required to complete a certain activity and start/finish dates of the activities related to it by preceding/proceeding relations. The expansion of scheduled time of an activity (especially when it is located on a critical path) results in a necessity of time compression in proceeding activities, which will ensure the scheduled project completion date. Such a compression, if possible, requires additional resources utilization resulting in project cost 
increase. The cost increase obviously causes negative and undesirable effects. It also can happen in an opposite direction - going beyond the budget results, at a certain stage, in a need to save in a further realization, which may cause delays in schedule. That is why, in most common cases, we have to deal with criteria conflicts when two objectives (time compression and cost cutting) are subjects to be reached. This kind of problem, referred to as time-cost trade-off [1], has been analyzed since the beginning of 1960s, just after CPM and PERT network approaches had been formulated.

In the most general form of completed project or milestone evaluation its success measure are deviations from schedule and budget. The uncertainty and risk, influencing all assumptions and system behaviour forecasts, cause the need to analyze the sensitivity of project schedule and budget and to insure the project against potential threats. Practical experience proves that reaction costs in execution phase with expected loss are usually incommensurably higher than additional cost of risk and alternative analysis during the planning phase. That is why the main emphasis is put on the complexity and profundity of a priori analyses during planning phase.

Decision alternative is an acceptable solution to the decision problem, different from other acceptable solutions. In project planning processes the decision alternative is usually referred to as mutually excluding project solutions, characterized by specific vectors of evaluation criteria. Project alternatives may be both repetitions of similar solutions used in the past with its adjustment and actualization to the current problem and products of team members creativity. The generation of innovative solutions is an essential problem of project planning related, for instance, to its realization in an unusual environment or research and development issues.

In decision making process chronology, the alternatives formulation proceeds just after decision criteria defining. Such an order allows for taking into account eventual system changes with respect to multi-criteria optimization model.

\section{PROBLEM FORMULATION}

We consider a project planning problem. Various resources can be used to complete project activities. In this paper we assume, that only a finite number of alternate resource allocations can be taken into account. For example, one, two or three workers can be employed to complete an activity. Thus, we face a discrete decision making problem, in which decision alternatives are defined by resource allocations.

The completion time depends on the resources allocated to the activity. We assume, that for each activity and for each alternate resource allocation, three completion time estimates are known: optimistic time, most probable time and pessimistic time. As activity times are uncertain, so the project completion time and project cost are uncertain as well.

The decision situation considered in this paper may be conceived as a problem $(A, X, E)$ where $A$ is a finite set of alternatives $a_{i}, i=1,2, \ldots, m, X$ is a finite set of criteria $X_{k}, k=1,2, \ldots, n$ and $E$ is a set of evaluations of alternatives with respect to criteria:

$$
\mathrm{E}=\left[\begin{array}{ccccc}
X_{11} & \cdots & X_{1 k} & \cdots & X_{1 n} \\
\vdots & \cdots & \vdots & \cdots & \vdots \\
X_{i 1} & \cdots & X_{i k} & \cdots & X_{i n} \\
\vdots & \cdots & \vdots & \cdots & \vdots \\
X_{m 1} & \cdots & X_{m k} & \cdots & X_{m n}
\end{array}\right]
$$

In our case, decision alternatives are evaluated with respect to two criteria: project completion time and total cost. Performances of each alternative with respect to the criteria are evaluated by distribution functions. The knowledge base used for the construction of these functions is obtained by using a simulation model. For each alternative, a series of simulations is carried out. The results are used for generating distributional evaluations of alternatives with respect to criteria.

\section{THE PROCEDURE}

Our approach consists in building global preferences on the set of resource allocations in two steps. First, alternatives are compared in relation to each criterion to model the partial preferences. Next, 
interactive procedure is used for selection of the most desirable alternative.

We use stochastic dominance [2] to model preferences with respect to each criterion. In this approach random variables are compared by pointwise comparison of their distribution functions assuming that larger values are preferred to smaller ones. In our problem criteria are minimized. Thus, to use stochastic dominance rules, we have to change the sign to the negative one for each criterion.

Let $F_{i k}(x)$ and $F_{j k}(x)$ be right-continuous cumulative distribution functions representing evaluations of $a_{i}$ and $a_{j}$ respectively over criterion $X_{k}$ :

$$
\begin{aligned}
& F_{i k}(x)=\operatorname{Pr}\left\{X_{i k} \leq x\right\} \\
& F_{j k}(x)=\operatorname{Pr}\left\{X_{j k} \leq x\right\}
\end{aligned}
$$

Definitions of the first and second stochastic dominance relations are as follows:

Definition 1. (FSD - First Stochastic Dominance)

$X_{i k}$ dominates $X_{j k}$ by FSD rule $\left(X_{i k} \succ_{\mathrm{FSD}} X_{j k}\right)$ if and only if

$F_{i k}(x) \neq F_{j k}(x)$ and

$H_{1}(x)=F_{i k}(x)-F_{j k}(x) \leq 0$ for $x \in \mathbf{R}$.

Definition 2. (SSD - Second Stochastic Dominance)

$X_{i k}$ dominates $X_{j k}$ by SSD rule $\left(X_{i k} \succ_{\mathrm{SSD}} X_{j k}\right)$ if and only if

$$
\begin{aligned}
F_{i k}(x) & \neq F_{j k}(x) \text { and } \\
H_{2}(x) & =\int_{-\infty}^{x} H_{1}(y) d y \leq 0 \text { for } x \in \mathbf{R}
\end{aligned}
$$

Hadar and Russel [3] have shown that the FSD rule is equivalent to the expected utility maximization rule for all decision makers preferring larger outcomes, while the SSD rule is equivalent to the expected utility maximization rule for risk-averse decision makers preferring larger outcomes. In this paper we assume that the decision maker is risk averse in relation to both criteria.

Once the relations between alternatives with respect to each criterion are identified, we are able to select efficient alternatives. We assume that alternative $a_{i}$ is efficient if and only if for no other alternative following condition is fulfilled [4]:

$\forall_{k=1, \ldots, n} X_{j k} \succ_{\mathrm{SD}} X_{i k}$

where $\succ_{\mathrm{SD}}$ stands for a stochastic dominance relation (FSD/SSD). Thus we assume that alternative $a_{i}$ is efficient if no other alternative dominates $a_{i}$ according to stochastic dominance rules with respect to all criteria.

We suggest using interactive technique INSDECM [5] for selection of the final solution. Each iteration includes the following steps:

- presentation of the data,

- $\quad$ asking the decision maker to provide preference information by specifying additional requirements,

- identification of alternatives satisfying decision maker's aspirations.

For each criterion the decision maker may choose one or more distribution characteristics to be presented (mean, median, standard deviation, quantilles). The best and the worst values of these measures attainable in the set of alternatives are identified and presented to the decision maker. Additional requirements are defined by specifying minimum or maximum values of distribution characteristics. Such restrictions, however, are in general not consistent with stochastic dominance rules ([6], [7]). We say that decision makers's requirement is not consistent with these rules if following conditions are fulfilled simultaneously:

- $\quad$ the evaluation of $a_{i}$ with respect to $X_{k}$ does not satisfy the requirement,

- the evaluation of $a_{j}$ with respect to $X_{k}$ satisfies the requirement,

$X_{i k} \succ_{\mathrm{SD}} X_{j k}$.

We propose to verify whether a constraint defined by the decision maker is consistent with stochastic dominance rules and to suggest methods of redefining constraint if inconsistency is found for any pair of projects. Let's assume that inconsistency has been verified for projects $a_{i}$ and $a_{j}$. Inconsistent 
constraint should be redefined in a way that results in accepting or rejecting both $a_{i}$ and $a_{j}$. The former can be achieved by making the constraint less restricted, the latter, by relaxing it.

INSDECM procedure operates as follows:

Generate the set of efficient alternatives $A$ '.

Assume $l=1, B_{l}=A^{\prime}$.

Ask the decision maker to specify distribution characteristics that should be presented during the conversational phase of the procedure.

Identify the best and the worst values of distribution characteristics attainable for $a_{i} \in B_{l}$; present the data to the decision maker.

Ask the decision maker whether he/she is satisfied with the data presented. If the answer is no, go to (3).

Ask the decision maker whether the worst values of distribution characteristics are satisfactory; if the answer is yes, go to (11).

Ask the decision maker to choose the characteristic, which should be improved and to specify minimum or maximum value of it.

Verify the consistency of the constraint specified by the decision maker with stochastic dominance rules. If the inconsistency is found, go to (9), else go to (10).

Present to the decision maker the ways in which the constraint can be redefined and ask him or her to choose one of the suggestions. If he or she does not accept any proposal, go to (7).

Generate $\mathrm{B}_{l+1}$ - the set of alternatives $a_{i} \in B_{l}$ satisfying decision maker's constraint. If $B_{l+1}=\varnothing$, notify the decision maker and go to (7), else assume $l=l+1$ and go to (4).

Present the list of considered alternatives to the decision maker. If he/she is able to choose the final solution, then end the procedure, else go to (7).

The procedure iterates until the decision maker is able to accept one of the considered projects as the final solution.

\section{NUMERICAL EXAMPLE}

An entrepreneur considers making a bid for equipment service for a leading producer on the market. The invitation for the project has been issued, as the previous contract is going to expire. The offer provides for a single service, but it is quite possible, that it will result in starting a long-term cooperation. As the contractor is a leading company on the market, so the success in tendering is considered to be of a primary importance. Thus, the overall goal of the entrepreneur is to win the tendering, even if the contract would not make a profit. The invitation for the project specifies all the tasks, that should be realized. The answer should specify the proposed price and the total time in which the project will be completed.

The project consists of eight time-consuming activities (fig. 1).

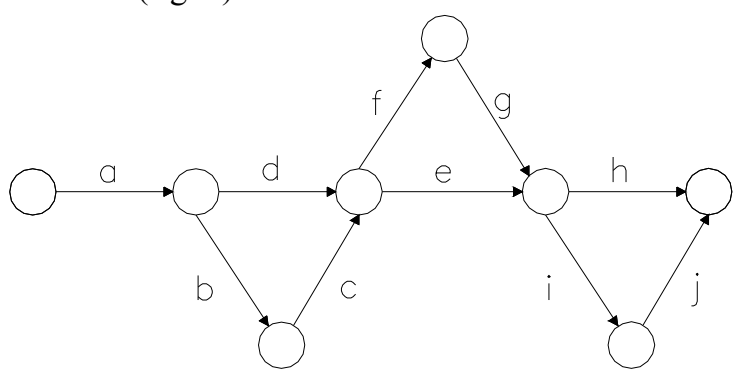

Figure 1. Project network

Two experienced employees may be engaged. While the first one (E1) is able to complete all the activities, the latter (E2) specializes in tasks, that are described by activities $b, c, e, h$ and $i$. Only one employee can be engaged for each activity.

As other contracts are also realized, so constraints related to the accessibility of employees have to be taken into account. Thus, when the answer for tendering is prepared, additional costs arising form tardiness of other projects have to be considered.

The decision maker is not sure how long each activity will take. However, three estimates for each activity has been obtained: optimistic time $(a)$, most probable time $(m)$ and pessimistic time $(b)$ (tab. 1). 
Table 1. List of activities

\begin{tabular}{|c|c|c|c|c|c|c|}
\hline Activity & $\begin{array}{c}\text { Optimistic time } \\
\text { [hours] }\end{array}$ & $\begin{array}{c}\text { Most probable } \\
\text { time [hours] }\end{array}$ & $\begin{array}{c}\text { Pessimistic } \\
\text { time [hours] }\end{array}$ & Employee & $\begin{array}{c}\text { Cost if the activity is } \\
\text { realized by employee } \\
\text { E1 [EUR/h] }\end{array}$ & $\begin{array}{c}\text { Cost if the activity is } \\
\text { realized by employee } \\
\text { E2 [EUR/h] }\end{array}$ \\
\hline$a$ & 9 & 12 & 18 & E1 & 30 & 0 \\
\hline$b$ & 6 & 8 & 12 & E1 or E2 & 30 & 45 \\
\hline$c$ & 3 & 4 & 6 & E1 or E2 & 35 & 50 \\
\hline$d$ & 9 & 12 & 18 & E1 & 30 & 0 \\
\hline$e$ & 6 & 8 & 12 & E1 or E2 & 45 & 65 \\
\hline$f$ & 3 & 4 & 6 & E1 & 30 & 0 \\
\hline$g$ & 3 & 4 & 6 & E1 & 25 & 45 \\
\hline$h$ & 18 & 24 & 36 & E1 or E2 & 30 & 65 \\
\hline$i$ & 9 & 12 & 18 & E1 or E2 & 50 & 0 \\
\hline$j$ & 6 & 8 & 12 & E1 & 40 & 0 \\
\hline
\end{tabular}

Table 2. The set of alternatives

\begin{tabular}{|c|c|c|c|c|c|c|c|c|c|c|c|c|c|c|c|c|}
\hline \multirow{2}{*}{ Activity } & \multicolumn{16}{|c|}{ Alternative } \\
\hline & $a_{1}$ & $a_{2}$ & $a_{3}$ & $a_{4}$ & $a_{5}$ & $a_{6}$ & $a_{7}$ & $a_{8}$ & $a_{9}$ & $a_{10}$ & $a_{11}$ & $a_{12}$ & $a_{13}$ & $a_{14}$ & $a_{15}$ & $a_{16}$ \\
\hline$a$ & E1 & E1 & E1 & E1 & E1 & E1 & E1 & E1 & E1 & E1 & E1 & E1 & E1 & E1 & E1 & E1 \\
\hline$b$ & E1 & E2 & E1 & E1 & E1 & E1 & E2 & E2 & E2 & $\mathrm{E} 2$ & E1 & E1 & E1 & E1 & E1 & E1 \\
\hline$c$ & E1 & E1 & E2 & E1 & E1 & E1 & E2 & E1 & E1 & E1 & E2 & E2 & E2 & E1 & E1 & E1 \\
\hline$d$ & E1 & E1 & E1 & E1 & E1 & E1 & E1 & E1 & E1 & E1 & E1 & E1 & E1 & E1 & E1 & E1 \\
\hline$e$ & E1 & E1 & E1 & E2 & E1 & E1 & E1 & E2 & E1 & E1 & E2 & E1 & E1 & E2 & E2 & E1 \\
\hline$f$ & E1 & E1 & E1 & E1 & E1 & E1 & E1 & E1 & E1 & E1 & E1 & E1 & E1 & E1 & E1 & E1 \\
\hline$g$ & E1 & E1 & E1 & E1 & E1 & E1 & E1 & E1 & E1 & E1 & E1 & E1 & E1 & E1 & E1 & E1 \\
\hline$h$ & E1 & E1 & E1 & E1 & E2 & E1 & E1 & E1 & E2 & E1 & E1 & $\mathrm{E} 2$ & E1 & E2 & E1 & E2 \\
\hline$i$ & E1 & E1 & E1 & E1 & E1 & E2 & E2 & E1 & E1 & E2 & E1 & E1 & E2 & E1 & E2 & E2 \\
\hline \multirow[t]{2}{*}{$j$} & E1 & E1 & E1 & E1 & E1 & E1 & E1 & E1 & E1 & E1 & E1 & E1 & E1 & E1 & E1 & E1 \\
\hline & $a_{17}$ & $a_{18}$ & $a_{19}$ & $a_{20}$ & $a_{21}$ & $a_{22}$ & $a_{23}$ & $a_{24}$ & $a_{25}$ & $a_{26}$ & $a_{27}$ & $a_{28}$ & $a_{29}$ & $a_{30}$ & $a_{31}$ & $a_{32}$ \\
\hline$a$ & E1 & E1 & E1 & E1 & E1 & E1 & E1 & E1 & E1 & E1 & E1 & E1 & E1 & E1 & E1 & E1 \\
\hline$b$ & E2 & E2 & E2 & E2 & E2 & E2 & E1 & E1 & E1 & E1 & E2 & E2 & E2 & E2 & E1 & E2 \\
\hline$c$ & E2 & E2 & E2 & E1 & E1 & E1 & E2 & E2 & E2 & E1 & E2 & E2 & E2 & E1 & E2 & E2 \\
\hline$d$ & E1 & E1 & E1 & E1 & E1 & E1 & E1 & E1 & E1 & E1 & E1 & E1 & E1 & E1 & E1 & E1 \\
\hline$e$ & E2 & E1 & E1 & E2 & E2 & E1 & E2 & E2 & E1 & E2 & E2 & E2 & E1 & E2 & E2 & E2 \\
\hline$f$ & E1 & E1 & E1 & E1 & E1 & E1 & E1 & E1 & E1 & E1 & E1 & E1 & E1 & E1 & E1 & E1 \\
\hline$g$ & E1 & E1 & E1 & E1 & E1 & E1 & E1 & E1 & E1 & E1 & E1 & E1 & E1 & E1 & E1 & E1 \\
\hline$h$ & E1 & E2 & E1 & E2 & E1 & E2 & E2 & E1 & E2 & E2 & E2 & E1 & E2 & E2 & E2 & E2 \\
\hline$i$ & E1 & E1 & E2 & E1 & E2 & E2 & E1 & E2 & E2 & E2 & E1 & E2 & E2 & E2 & E2 & E2 \\
\hline$j$ & E1 & E1 & E1 & E1 & E1 & E1 & E1 & E1 & E1 & E1 & E1 & E1 & E1 & E1 & E1 & E1 \\
\hline
\end{tabular}


Differences in the labour costs arise from the fact, that at this moment employees are engaged in other projects. Thus, the cost of the project has to be increased by the cost of delays of other projects.

Taking into account the information about possible employees assignments, the set of alternate resource allocations (alternatives) has been generated (tab. 2).

To solve the problem, simulations have been run for each alternative and each project. We used MSEXCEL assuming that the uncertainty in activity times can be described by a triangular distributions. Results of simulation experiments are presented in table 3 .

In the second stage we generate the set of efficient alternatives $A^{\prime}$. We compare evaluations of alternatives employing stochastic dominance rules. The efficient set consists of 18 alternatives:

$A^{\prime}=\left\{a_{1}, a_{2}, a_{3}, a_{6}, a_{7}, a_{9}, a_{10}, a_{12}, a_{13}, a_{15}, a_{17}, a_{18}\right.$, $\left.a_{19}, a_{20}, a_{21}, a_{23}, a_{27}, a_{28}\right\}$
For example alternative $a_{4}$ is dominated by alternative $a_{2}$, as:

$X_{21} \succ_{\mathrm{SSD}} X_{41}$ and $X_{22} \succ_{\mathrm{SSD}} X_{42}$

Finally, interactive procedure is used for generating the solution of the problem.

$l=1, B_{1}=A^{\prime}$.

Iteration 1

The decision maker specified distribution characteristics to be presented during the conversational phase of the procedure:

- criterion $X_{1}$ : mean and probability that the completion time will not exceed 95 hours,

- criterion $X_{2}$ : mean and probability that the cost will not exceed 4000 EUR.

The best and the worst values of distribution characteristics were presented to the decision maker (tab. 4)

Table 3. Results of simulation experiments

\begin{tabular}{|c|c|c|c|c|c|c|c|c|c|}
\hline \multirow{2}{*}{$\begin{array}{l}\text { Alter- } \\
\text { native }\end{array}$} & \multicolumn{2}{|c|}{ Time } & \multicolumn{2}{|c|}{ Cost } & \multirow{2}{*}{$\begin{array}{l}\text { Alter- } \\
\text { native }\end{array}$} & \multicolumn{2}{|c|}{ Time } & \multicolumn{2}{|c|}{ Cost } \\
\hline & Mean & $\begin{array}{l}\text { Standard } \\
\text { deviation }\end{array}$ & Mean & $\begin{array}{l}\text { Standard } \\
\text { deviation }\end{array}$ & & Mean & $\begin{array}{l}\text { Standard } \\
\text { deviation }\end{array}$ & Mean & $\begin{array}{l}\text { Standard } \\
\text { deviation }\end{array}$ \\
\hline$a_{1}$ & 105,17 & 4,94 & 3629,69 & 177,23 & $a_{17}$ & 84,35 & 4,59 & 3999,68 & 190,11 \\
\hline$a_{2}$ & 96,49 & 4,92 & 3758,78 & 173,92 & $a_{18}$ & 70,96 & 4,20 & 4207,50 & 212,96 \\
\hline$a_{3}$ & 100,44 & 4,96 & 3692,82 & 167,10 & $a_{19}$ & 79,27 & 4,41 & 4035,38 & 199,33 \\
\hline$a_{4}$ & 96,85 & 4,95 & 3807,21 & 186,95 & $a_{20}$ & 66,60 & 3,97 & 4319,55 & 207,13 \\
\hline$a_{5}$ & 83,41 & 4,49 & 4024,44 & 208,98 & $a_{21}$ & 74,86 & 4,49 & 4132,47 & 196,53 \\
\hline$a_{6}$ & 91,54 & 4,58 & 3835,99 & 189,40 & $a_{22}$ & 87,76 & 4,84 & 4349,29 & 218,83 \\
\hline$a_{7}$ & 92,45 & 4,82 & 3834,51 & 180,32 & $a_{23}$ & 71,06 & 4,30 & 4266,35 & 218,02 \\
\hline$a_{8}$ & 88,43 & 4,69 & 3944,54 & 187,88 & $a_{24}$ & 79,40 & 4,57 & 4069,64 & 201,48 \\
\hline$a_{9}$ & 74,51 & 4,27 & 4148,37 & 212,40 & $a_{25}$ & 91,92 & 4,94 & 4280,08 & 216,85 \\
\hline$a_{10}$ & 82,86 & 4,45 & 3961,77 & 186,43 & $a_{26}$ & 88,21 & 4,70 & 4395,29 & 222,66 \\
\hline$a_{11}$ & 92,89 & 4,95 & 3869,55 & 185,09 & $a_{27}$ & 63,08 & 4,05 & 4390,83 & 224,24 \\
\hline$a_{12}$ & 78,83 & 4,40 & 4080,17 & 214,30 & $a_{28}$ & 71,37 & 4,27 & 4207,90 & 205,23 \\
\hline$a_{13}$ & 87,65 & 4,78 & 3889,67 & 184,01 & $a_{29}$ & 84,14 & 4,53 & 4435,24 & 222,84 \\
\hline$a_{14}$ & 75,18 & 4,47 & 4193,57 & 211,86 & $a_{30}$ & 79,41 & 4,74 & 4526,85 & 237,88 \\
\hline$a_{15}$ & 83,61 & 4,68 & 4013,77 & 193,98 & $a_{31}$ & 83,89 & 4,61 & 4468,38 & 228,42 \\
\hline$a_{16}$ & 96,29 & 4,94 & 4227,24 & 211,08 & $a_{32}$ & 76,16 & 4,56 & 4592,72 & 220,43 \\
\hline
\end{tabular}


Table 4. Data presented to the decision maker in iteration 1

\begin{tabular}{|c|c|c|c|c|}
\hline \multirow{2}{*}{} & \multicolumn{2}{|c|}{$X_{1}$} & \multicolumn{2}{|c|}{$X_{2}$} \\
\cline { 2 - 5 } & mean & $\operatorname{Pr}\left\{X_{i 1} \leq 95\right\}$ & mean & $\operatorname{Pr}\left\{X_{i 2} \leq 4000\right\}$ \\
\hline the best value & 63,08 & 1,000 & 3629,69 & 0,980 \\
\hline the worst value & 105,17 & 0,014 & 4390,83 & 0,035 \\
\hline
\end{tabular}

Table 5. Data presented to the decision maker in iteration 2

\begin{tabular}{|c|c|c|c|c|}
\hline & \multicolumn{2}{|c|}{$X_{1}$} & \multicolumn{2}{|c|}{$X_{2}$} \\
\cline { 2 - 5 } & mean & $\operatorname{Pr}\left\{X_{i 1} \leq 95\right\}$ & mean & $\operatorname{Pr}\left\{X_{i 2} \leq 4000\right\}$ \\
\hline the best value & 63,08 & 1,000 & 3889,67 & 0,731 \\
\hline the worst value & 87,65 & 0,922 & 4390,83 & 0,035 \\
\hline
\end{tabular}

Table 6. Data presented to the decision maker in iteration 3

\begin{tabular}{|c|c|c|c|c|}
\hline \multirow{2}{*}{} & \multicolumn{2}{|c|}{$X_{1}$} & \multicolumn{2}{c|}{$X_{2}$} \\
\cline { 2 - 5 } & mean & $\operatorname{Pr}\left\{X_{i 1} \leq 95\right\}$ & mean & $\operatorname{Pr}\left\{X_{i 2} \leq 4000\right\}$ \\
\hline the best value & 82,86 & 0,997 & 3889,67 & 0,731 \\
\hline the worst value & 87,65 & 0,922 & 3999,68 & 0,508 \\
\hline
\end{tabular}

The decision maker was not satisfied with the worst values of distribution characteristics and specified additional requirement: "probability that the completion time will not exceed 95 should not be less than 0,90":

$\operatorname{Pr}\left\{X_{i 1} \leq 95\right\} \geq 0,90$.

The restriction was consistent with stochastic dominance rules.

The set of alternatives satisfying the restriction was generated:

$B_{2}=\left\{a_{9}, a_{10}, a_{12}, a_{13}, a_{15}, a_{17}, a_{18}, a_{19}, a_{20}, a_{21}, a_{23}\right.$, $\left.a_{27}, a_{28}\right\}$

$l=2$.

Iteration 2

The best and the worst values of distribution characteristics were presented to the decision maker (tab. 5).

The decision maker was not satisfied with the worst values of distribution characteristics and specified additional requirement: "probability that the cost will not exceed 4000 should not be less than 0,50 ":

$\operatorname{Pr}\left\{X_{i 2} \leq 4000\right\} \geq 0,50$.
The restriction was consistent with stochastic dominance rules.

The set of alternatives satisfying the restriction was generated:

$B_{3}=\left\{a_{10}, a_{13}, a_{17}\right\}$

$l=3$.

Iteration 3

The best and the worst values of distribution characteristics were presented to the decision maker (tab. 6).

The decision maker was satisfied with the worst values of distribution characteristics

Alternatives satisfying restrictions were presented to the decision maker (tab. 7).

The decision maker selected alternative $a_{13}$ as a final solution

As a result, it was decided to prepare an answer to the tendering assuming that employee E1 would be engaged for activities $a, b, d, e, f, g, h$ and $j$, while employee E2 for activities $c$ and $i$. 
Table 7. Alternatives satisfying restrictions specified by the decision maker

\begin{tabular}{|c|c|c|c|c|}
\hline \multirow{2}{*}{ Alternative } & \multicolumn{2}{|c|}{$X_{1}$} & \multicolumn{2}{|c|}{$X_{2}$} \\
\cline { 2 - 5 } & mean & $\operatorname{Pr}\left\{X_{i 1} \leq 95\right\}$ & mean & $\operatorname{Pr}\left\{X_{i 2} \leq 4000\right\}$ \\
\hline$a_{10}$ & 82,86 & 0,997 & 3961,77 & 0,594 \\
\hline$a_{13}$ & 87,65 & 0,922 & 3889,67 & 0,731 \\
\hline$a_{17}$ & 84,35 & 0,987 & 3999,68 & 0,508 \\
\hline
\end{tabular}

\section{CONCLUSIONS}

In the paper a procedure for time-cost trade-off problem in project planning was presented. The procedure uses simulation model and interactive technique.

Interactive approach is one of the leading methodologies in multiple criteria decision making. Several motivations have been mentioned for implementing this approach. It is usually pointed out that limited amount of a priori preference information is required from the decision maker as compared to other techniques. The interactive procedure may be considered as a learning process. Observing the results of succeeding iterations of the procedure the decision maker extends his/her knowledge of the decision problem. On the other hand, as the decision maker actively participated in all phases of problem solving procedure, the decision maker puts much reliance on the final solution that is obtained. As a result, the solution of the procedure has a better chance of being implemented.

The technique proposed in this paper may be useful for various types of problems in which uncertain outcomes are compared. It has been designed for problems with up to moderate number of discrete actions (not more than hundreds) and can be applied also in such areas as, for example, inventory models, evaluation of investment projects, production process control, and many others.

\section{REFERENCES}

[1] De, P., Dunne, E.J., Ghosh, J.B. \& Wells, Ch.E. (1995), The discrete time-cost tradeoff problem revisited, European Journal of Operational Research, Vol. 81, 225-238.

[2] Zaras, K. \& Martel, J.M. (1994) Multiattribute analysis based on stochastic dominance, Models and Experiments in Risk and Rationality, Eds.: Munier, B. \& Machina M.J. Kluwer Academic Publishers, Dordrecht, 225-248.

[3] Hadar, J. \& Russel, W.R. (1969). Rules for Ordering Uncertain Prospects. The American Economic Review, Vol. 59, 25-34.

[4] Huang, C.C., Kira, D. \& Vertinsky, I. (1978). Stochastic dominance rules for multiattribute utility functions. Review of Economic Studies, Vol. 41, 611-616.

[5] Nowak, M. (2006). INSDECM - an interactive procedure for stochastic multicriteria decision problems. European Journal of Operational Research, Vol. 175, 1413-1430.

[6] Ogryczak, W. \& Ruszczyński, A. (1999). From stochastic dominance to mean-risk models: Semideviations as risk measures. European Journal of Operational Research, Vol. 116, 33-50.

[7] Ogryczak, W. \& Ruszczyński, A. (2001). On consistency of stochastic dominance and meansemideviation models. Mathematical Programming, Vol. 89, 217-232. 